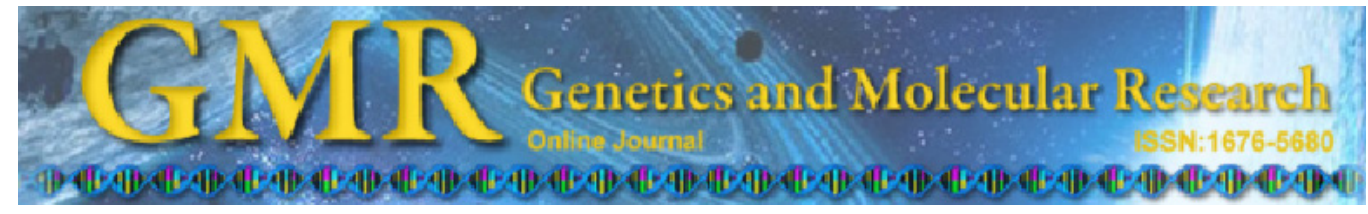

\title{
Lack of an association between $T S C$ gene Arg904GIn polymorphisms and essential hypertension risk based on a meta-analysis
}

\author{
F. Zhang ${ }^{1 *}$, Y. Yang ${ }^{2 *}$, D. Hu², H. Lei ${ }^{2}$ and Y. Wang ${ }^{3}$ \\ ${ }^{1}$ School of Public Health, Chongqing Medical University, Chongqing, China \\ ${ }^{2}$ Department of Cardiology, First Affiliated Hospital, \\ Chongqing Medical University, Chongqing, China \\ ${ }^{3}$ China Network of Effective Health Care Research Consortium, \\ Chongqing Medical University, Chongqing, China \\ *These authors contributed equally to this study. \\ Corresponding author: Y. Wang \\ E-mail: ebm_cq@163.com
}

Genet. Mol. Res. 11 (3): 3511-3517 (2012)

Received November 10, 2011

Accepted March 27, 2012

Published September 26, 2012

DOI http://dx.doi.org/10.4238/2012.September.26.7

ABSTRACT. Although there have been several studies investigating a possible association between essential hypertension and TSC gene Arg904Gln polymorphisms, the results have been inconsistent. We conducted a meta-analysis of four case-control studies (one study in Europe and three studies in Asia), including 1811 essential hypertension cases and 1381 controls. The pooled results showed no significant associations between any of these polymorphisms and essential hypertension (allele Arg vs allele Gln: odds ratio $(\mathrm{OR})=0.94,95 \%$ confidence interval $(95 \% \mathrm{CI})$ $=0.70-1.27)$, additive genetic model (Arg/Arg vs $\mathrm{Gln} / \mathrm{Gln}: \mathrm{OR}=0.98$, $95 \% \mathrm{CI}=0.43-2.23)$, dominant genetic model (Arg/Arg $+\mathrm{Arg} / \mathrm{Gln} v_{s} \mathrm{Gln} /$ Gln: $\mathrm{OR}=0.97,95 \% \mathrm{CI}=0.43-2.21$ ), and recessive genetic model (Arg/ $\operatorname{Arg} v s \mathrm{Arg} / \mathrm{Gln}+\mathrm{Gln} / \mathrm{Gln}: \mathrm{OR}=1.03,95 \% \mathrm{CI}=0.45-2.35)$. Based on the results of our meta-analysis, we conclude that the TSC gene Arg904Gln polymorphism is not associated with essential hypertension risk.

Key words: TSC; $\operatorname{Arg} 904 \mathrm{Gln}$; Polymorphisms; Essential hypertension; Meta-analysis 


\section{INTRODUCTION}

Essential hypertension is a complex, multi-factorial, and polygenic disease; it is one of the most important risk factors for cardiovascular disease, leading to over 450,000 deaths in the United States of America in 2004 (Capewell et al., 2010). Essential hypertension is caused by genetic and environmental factors. Many risk factors have been associated with essential hypertension, including age, gender, obesity, and excess salt intake (Stanto et al., 1982). Recently, genetic investigations have revealed several genetic variants that are candidates in the pathogenesis of hypertension (Johnson et al., 2011; Hasi et al., 2011; Fu et al., 2011). Current studies suggest that the thiazide-sensitive $\mathrm{Na}-\mathrm{Cl}$ cotransporter (TSC) gene plays an important role on the risk of essential hypertension in diverse ethnic groups (Melander et al., 2000; Song et al., 2001; Glorioso et al., 2001; Matsuo et al., 2004; Zhan et al., 2007; Wang et al., 2008). The TSC gene is located on chromosome 16q13, consisting of 26 exons that encode 1021-amino acid residues (Plotkin et al., 1996). Among the SNPs of the TSC gene, Arg904Gln is at amino acid position 904 of the thiazide-sensitive NaCl-cotransporter, the $\mathrm{G} \rightarrow \mathrm{A}$ mutation in exon 23 of the TSC gene (G2736A), which leads to a replacement of Arg by Gln (Melander et al., 2000). Compared with other SNPs of the TSC gene, such as Gly264Ala and C1420T (Melander et al., 2000 ), it is reported more frequently as associated with essential hypertension risk.

Several studies have evaluated the association between TSC gene Arg904Gln polymorphisms and essential hypertension risk. However, the results remain somewhat contradictory and inconclusive. To overcome the limitations of individual studies and to understand the real situation, we made a meta-analysis of four case-control studies.

\section{MATERIAL AND METHODS}

\section{Search strategy}

We searched the PubMed and Embase database for studies published up to June of 2011, by using items: "thiazide-sensitive Na-Cl cotransporter gene", "TSC gene", "Arg904Gln", "G2736A", "hypertension", and "blood pressure". We also sought additional studies by reviewing the reference lists of included articles, conference abstracts, and relevant bibliographies.

\section{Selection criteria}

Two reviewers (Zhang F and Yang Y) reviewed all citations and retrieved literature by titles/abstracts and whole texts. Those studies were selected by meeting the following criteria: 1 ) the publication was a case-control study referring to association between the TSC gene and the risk for essential hypertension; 2) the papers must offer sample size, distribution of alleles, genotypes, or other information that can help us infer the results; 3 ) when multiple publications reported on the same or overlapping data, we used the most recent or largest population as recommended by Little et al. (2002), and 4) we limited the publication languages to English and Chinese.

\section{Data extraction}

Data were extracted from each study by two reviewers (Zhang F and Yang Y), independently according to the pre-specified selection criteria. Extraction forms of two reviewers 
were compared. Disagreements were discussed. Decisions were made by consensus or by involving a third reviewer. The following information was extracted from the studies: first author, publishing year, studied polymorphisms, ethnicity of subjects, source of controls, and distribution of alleles and genotypes in case and control groups.

\section{Statistical analysis}

Crude odds ratios (ORs) with their 95\% confidence intervals (95\%CIs) for alleles and genotypes were used to assess the strength of association between TSC gene Arg904Gln polymorphisms and essential hypertension risk. The pooled ORs were performed for the allele contrasts, additive genetic model, dominant genetic model, and recessive genetic model. Heterogeneity assumption was assessed by the chi-square-based Q-test and the I-squared test. The heterogeneity was not considered significant when $\mathrm{I}^{2}<50 \%$. With lack of heterogeneity among studies, the pooled OR estimate of each study was calculated by the fixed-effect model (Wang et al., 2008). Otherwise, the random-effect model was used (Matsuo et al., 2004; Zhan et al., 2007). Departure of frequencies of TSC gene Arg904Gln polymorphisms from expectation according to Hardy-Weinberg equilibrium (HWE) was assessed by the chisquare test in controls. Funnel plots were used to assess the possibility of publication bias.

The Cochrane Collaboration meta-analysis software, Review Manager 5.0, was used for analysis. A P value of 0.05 for any test or model was considered to be statistically significant.

\section{RESULTS}

\section{Eligible studies}

After careful examination according to the inclusion criteria, our final pool of eligible studies included four case-control studies with 1811 cases and 1381 controls. As shown in Table 1, there was one study involving European subjects and three studies involving Asian subjects (two in Chinese including 3 ethnic groups and one in Japanese). Almost all of the cases were histologically confirmed. Controls were mainly healthy individuals of the same population, which were matched for age and ethnicity. The genotype distribution in the controls of all studies was in agreement with HWE except for one study (Zhan et al., 2007).

\begin{tabular}{|c|c|c|c|c|c|c|c|c|}
\hline \multirow[t]{2}{*}{ Author } & \multirow[t]{2}{*}{ Ethnicity } & \multirow[t]{2}{*}{ Source of control } & \multicolumn{3}{|c|}{ Case } & \multicolumn{3}{|c|}{ Control } \\
\hline & & & AA & $\mathrm{AG}$ & GG & AA & $\mathrm{AG}$ & GG \\
\hline \multirow[t]{2}{*}{ Wang et al., 2008} & Kazaks & Population-based & 472 & 117 & 4 & 250 & 53 & 1 \\
\hline & Uyghurs & Population-based & 278 & 61 & 0 & 262 & 68 & 7 \\
\hline Zhan et al., 2007 & Han & Population-based & 167 & 22 & 1 & 83 & 10 & 1 \\
\hline Matsuo et al., 2004 & Japanese & Hospital-based & 332 & 52 & 2 & 338 & 33 & 0 \\
\hline Melander et al., 2000 & Europe & Population-based & 239 & 48 & 5 & 210 & 54 & 0 \\
\hline
\end{tabular}

\section{Quantitative synthesis}

The main results of this meta-analysis are shown in Table 2 and the heterogeneity tests 
are shown in Figures 1-4. Overall, no significant associations were found for the allele contrast (allele Arg vs allele Gln: $\mathrm{OR}=0.94,95 \% \mathrm{CI}=0.70-1.27$ ), additive genetic model (Arg/Arg $v_{s} \mathrm{Gln} / \mathrm{Gln}$ : OR $\left.=0.98,95 \% \mathrm{CI}=0.43-2.23\right)$, dominant genetic model $(\mathrm{Arg} / \mathrm{Arg}+\mathrm{Arg} / \mathrm{Gln} v s$ $\mathrm{Gln} / \mathrm{Gln}: \mathrm{OR}=0.97,95 \% \mathrm{CI}=0.43-2.21)$, or recessive genetic model $(\mathrm{Arg} / \mathrm{Arg} v s \mathrm{Arg} / \mathrm{Gln}+$ $\mathrm{Gln} / \mathrm{Gln}: \mathrm{OR}=1.03,95 \% \mathrm{CI}=0.45-2.35)$.

Table 2. Summary of odds ratio (OR) and 95\% confidence interval (95\%CI) of the TSC gene Arg904Gln polymorphism and essential hypertension risk.

\begin{tabular}{lccc}
\hline Polymorphism & OR & $95 \%$ C & P \\
\hline A $v s$ G* & 0.94 & $0.70-1.27$ & 0.7 \\
AA $v s$ GG & 0.98 & $0.43-2.23$ & 0.96 \\
AA+AG $v s$ GG & 0.97 & $0.43-2.21$ & 0.94 \\
AA $v s$ AG+GG & 1.03 & $0.45-2.35$ & 0.94 \\
\hline
\end{tabular}

*Estimates for a random-effect model.

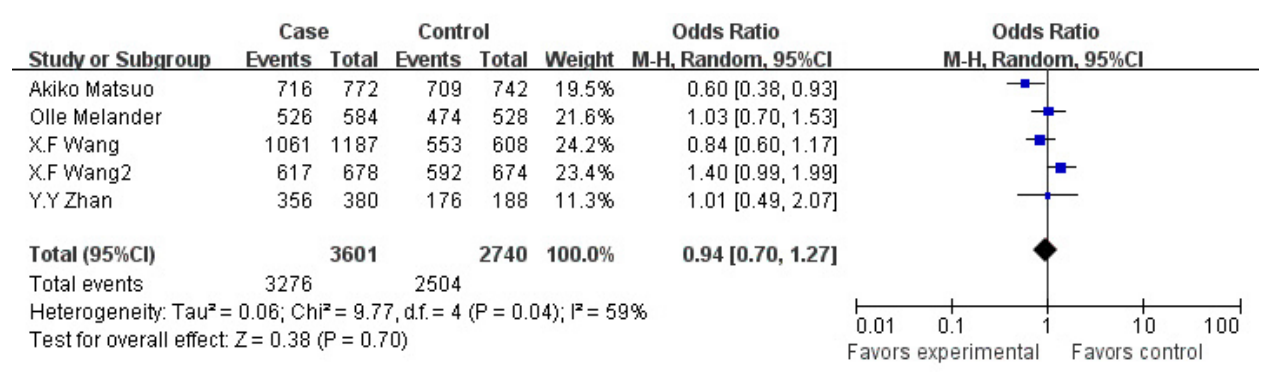

Figure 1. Forest plots of the allele contrast. $\mathrm{M}-\mathrm{H}=$ Mantel-Haenszel estimator; $95 \% \mathrm{CI}=$ confidence interval at $95 \%$; d.f. $=$ degrees of freedom.

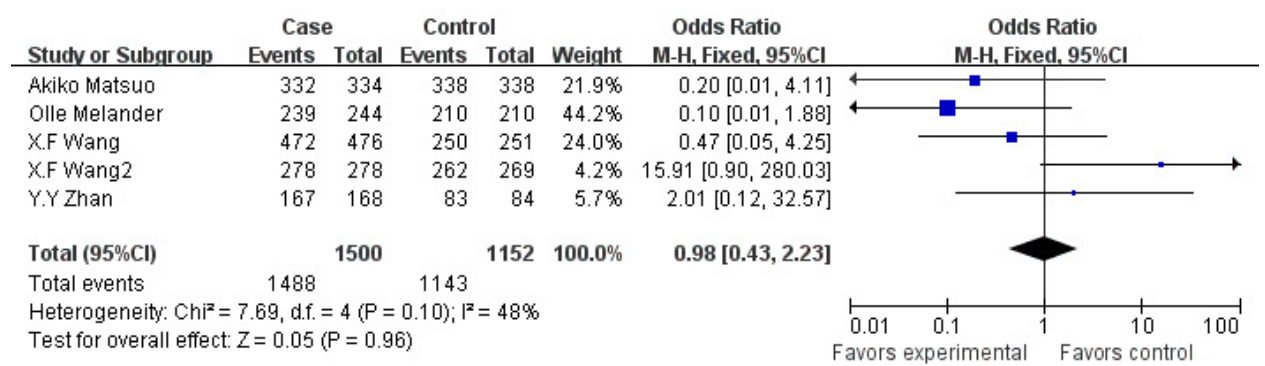

Figure 2. Forest plots of additive genetic model. For abbreviations, see legend to Table 1.

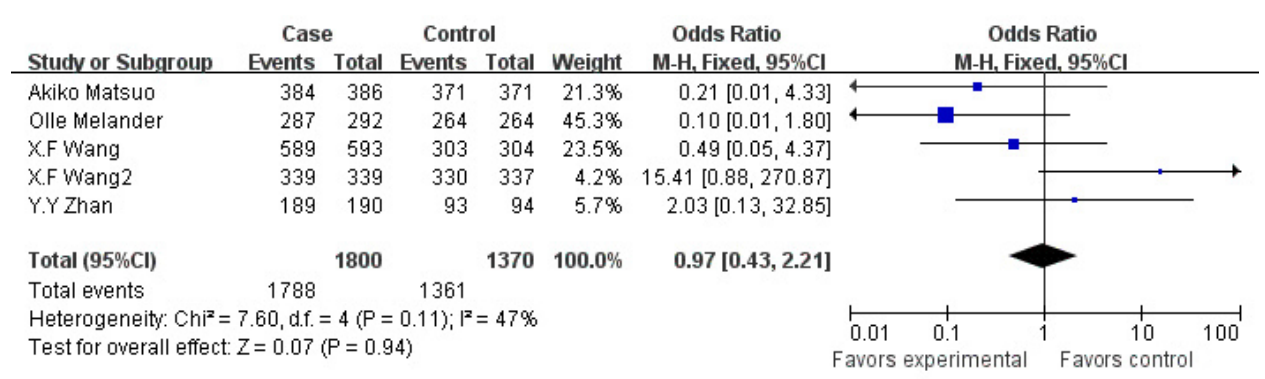

Figure 3. Forest plots of dominant genetic model. For abbreviations, see legend to Table 1. 


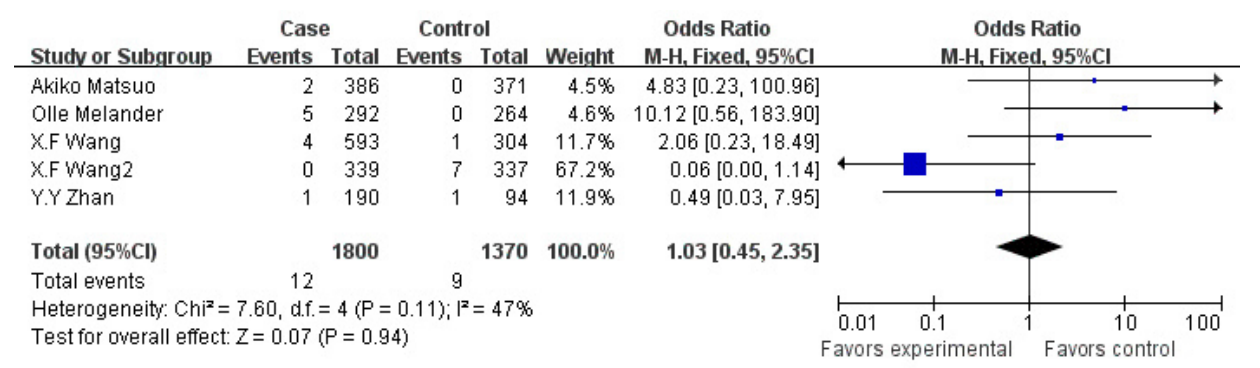

Figure 4. Forest plots of recessive genetic model. For abbreviations, see legend to Table 1.

\section{Sensitivity analysis}

Sensitivity analyses were conducted to determine whether modification of the inclusion criteria of the meta-analysis affected the final results. These were carried out by limiting the meta-analysis to studies conforming to HWE and altering corresponding statistic variables and analysis models. Additional sensitivity analyses indicated that the point estimate and $95 \% \mathrm{CI}$ for the OR were not significantly altered with the exclusion of any individual study. We concluded that our results were statistically robust.

\section{Bias diagnosis}

Funnel plots were made to access the publication bias of literatures. As shown in Figure 5 , the shape of the funnel plot revealed relatively symmetry.
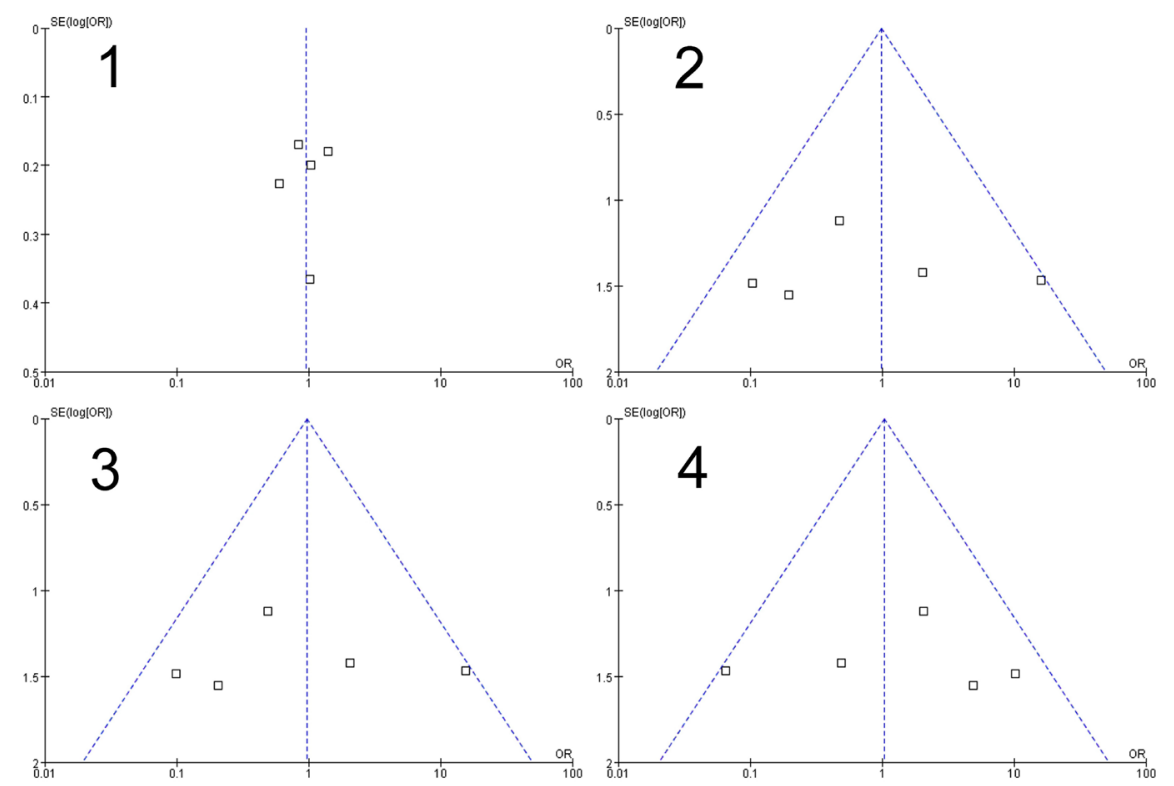

Figure 5. Funnel plots: Funnel plot 1 for allele comparison; Funnel plot 2 for additive genetic model; Funnel plot 3 for dominant genetic model; Funnel plot 4 for recessive genetic model. 


\section{DISCUSSION}

Hypertension is a common disease caused by polygenic factors combined with environment. Many candidate genes have been reported to be involved in essential hypertension susceptibility, including TSC, NEDD4L (Luo et al., 2009), CYP4F2 (Ward et al., 2008), EMILIN 1 (Shimodaira et al., 2010), ATP2B1 (Tabara et al., 2010), apelin-AGTRL1 system (Niu et al., 2010), and so on. Several studies suggest that TSC plays a major role in sodium and chloride re-absorption in the distal convoluted tubule, accounting for a significant proportion of the total renal sodium re-absorption (Plotkin et al., 1996; Matsuo et al., 2004). TSC is a target of the diuretic effect of thiazides and is an established antihypertensive drug in the management of uncomplicated hypertension. Mutations in the human TSC (hTSC) gene affect blood pressure regulation, as seen in Gitelman's syndrome (Melander et al., 2000), which is a disease characterized by sodium wasting and low blood pressure. These characteristics validated the TSC gene as a logical candidate gene for hypertension susceptibility. In order to obtain persuasive results, we did this meta-analysis to evaluate the association of TSC gene Are904Gln polymorphisms with essential hypertension susceptibility.

Melander et al. (2000) found that the Arg904Gln polymorphism is significantly associated with hypertension in the Swedish population. Gln904 homo-zygotes were overrepresented in hypertensive patients compared with normotensive subjects ( 5 of 292 vs 0 of 264; $\mathrm{P}=0.03$ ). Matsuo et al. (2004) showed that the Arg904Gln (G2736A) polymorphism is significantly associated with prevalence of hypertension $(\mathrm{P}<0.04)$, and the estimated OR was $1.8(95 \% \mathrm{CI}=$ 1.1-3.0). However, the other two studies were performed on the Chinese Han ethnic group, and other two minorities - Kazaks and Uyghurs (Wang et al., 2008). In Han and Uyghurs, there was no association between the Arg904Gln polymorphism and hypertension risk, but in the minority Kazaks, the results were different from those of the Swedish and Japanese populations. A stronger trend of $904 \mathrm{Gln}$ allele in controls than hypertensives $(\mathrm{P}=0.058)$ was observed, and the greater prevalence of the $904 \mathrm{Gln}$ carrier genotype reached significance $(\mathrm{P}=0.015)$.

Combining all of the studies, our meta-analysis found no significant associations between Arg904Gln polymorphisms and essential hypertension for the allele contrast (allele Arg vs allele Gln: $\mathrm{OR}=0.94,95 \% \mathrm{CI}=0.70-1.27)$, additive genetic model $(\mathrm{Arg} / \mathrm{Arg} v s \mathrm{Gln} / \mathrm{Gln}$ : $\mathrm{OR}=0.98,95 \% \mathrm{CI}=0.43-2.23)$, dominant genetic model $(\mathrm{Arg} / \mathrm{Arg}+\mathrm{Arg} / \mathrm{Gln} v s \mathrm{Gln} / \mathrm{Gln}: \mathrm{OR}$ $=0.97,95 \% \mathrm{CI}=0.43-2.21)$, or recessive genetic model $(\mathrm{Arg} / \mathrm{Arg} v s \mathrm{Arg} / \mathrm{Gln}+\mathrm{Gln} / \mathrm{Gln}: \mathrm{OR}$ $=1.03,95 \% \mathrm{CI}=0.45-2.35)$.

A recent study on association of TSC gene variants and hypertension in Mongolian and Han populations (Chang et al., 2011) included 385 unrelated Mongolian herdsmen and 523 Han farmers, and found significant differences between the genotype and allele frequencies of rs13306673 between the essential hypertension group and the control group in the Han population and significant associations between the rs7204044 variant and essential hypertension in both the Mongolian and Han ethnic groups. However, the mechanisms by which rs13306673 and rs 7204044 might contribute to essential hypertension are currently unknown. So we do not know whether there is a relationship between it and the Arg904Gln or not. More research should be done, and larger data sets should be analyzed.

There are some limitations in our review. First, the bias between the ethnic groups was obvious and the combined studies were few, only four studies referring to five ethnic groups. Second, the controls of one study were hospital-based normal individuals or patients 
of other diseases, which did not possess adequate representation (Matsuo et al., 2004). Third, the languages were limited to English and Chinese for the systematic review. Furthermore, the TSC gene contains many more polymorphisms than those mentioned in our review. Given the limited evidence available on other TSC gene polymorphisms, this article was restricted to the TSC gene Arg904Gln polymorphism.

Conclusively, our review suggests that TSC gene Arg904Gln polymorphisms are not associated with essential hypertension risk. Large well-designed and multi-center epidemiological studies will be necessary to check genetic factors in diverse ethnicities.

\section{REFERENCES}

Capewell S, Ford ES, Croft JB, Critchley JA, et al. (2010). Cardiovascular risk factor trends and potential for reducing coronary heart disease mortality in the United States of America. Bull. World Health Organ. 88: 120-130.

Chang PY, Zhao LG and Su XL (2011). Association of TSC gene variants and hypertension in Mongolian and Han populations. Genet. Mol. Res. 10: 902-909.

Fu L, Zhao Y, Wu X, Liu H, et al. (2011). CYP7A1 genotypes and haplotypes associated with hypertension in an obese Han Chinese population. Hypertens. Res. 34: 722-727.

Glorioso N, Filigheddu F, Troffa C, Soro A, et al. (2001). Interaction of $\alpha(1)-\mathrm{Na}, \mathrm{K}-\mathrm{ATP}$ ase and Na,K,2Cl-cotransporter genes in human essential hypertension. Hypertension 38: 204-209.

Hasi T, Hao L, Yang L and Su XL (2011). Acetaldehyde dehydrogenase 2 SNP rs671 and susceptibility to essential hypertension in Mongolians: a case control study. Genet. Mol. Res. 10: 537-543.

Johnson AD, Newton-Cheh C, Chasman DI, Ehret GB, et al. (2011). Association of hypertension drug target genes with blood pressure and hypertension in 86,588 individuals. Hypertension 57: 903-910.

Little J, Bradley L, Bray MS, Clyne M, et al. (2002). Reporting, appraising, and integrating data on genotype prevalence and gene-disease associations. Am. J. Epidemiol. 156: 300-310.

Luo F, Wang Y, Wang X, Sun K, et al. (2009). A functional variant of NEDD4L is associated with hypertension, antihypertensive response, and orthostatic hypotension. Hypertension 54: 796-801.

Matsuo A, Katsuya T, Ishikawa K, Sugimoto K, et al. (2004). G2736A polymorphism of thiazide-sensitive Na-Cl cotransporter gene predisposes to hypertension in young women. J. Hypertens. 22: 2123-2127.

Melander O, Orho-Melander M, Bengtsson K, Lindblad U, et al. (2000). Genetic variants of thiazide-sensitive NaClcotransporter in gitelman's syndrome and primary hypertension. Hypertension 36: 389-394.

Niu W, Wu S, Zhang Y, Li W, et al. (2010). Validation of genetic association in apelin-AGTRL1 system with hypertension in a larger Han Chinese population. J. Hypertens. 28: 1854-1861.

Plotkin MD, Kaplan MR, Verlander JW, Lee WS, et al. (1996). Localization of the thiazide sensitive Na-Cl cotransporter, rTSC1 in the rat kidney. Kidney Int. 50: 174-183.

Shimodaira M, Nakayama T, Sato N, Naganuma T, et al. (2010). Association study of the elastin microfibril interfacer 1 (EMILIN1) gene in essential hypertension. Am. J. Hypertens. 23: 547-555.

Song Y, Herrera VL, Filigheddu F, Troffa C, et al. (2001). Non-association of the thiazide-sensitive Na,Cl-cotransporter gene with polygenic hypertension in both rats and humans. J. Hypertens. 19: 1547-1551.

Stanton JL, Braitman LE, Riley AM Jr, Khoo CS, et al. (1982). Demographic, dietary, life style, and anthropometric correlates of blood pressure. Hypertension 4: III135-III142.

Tabara Y, Kohara K, Kita Y, Hirawa N, et al. (2010). Common variants in the ATP2B1 gene are associated with susceptibility to hypertension: the Japanese Millennium Genome Project. Hypertension 56: 973-980.

Wang XF, Lin RY, Wang SZ, Zhang LP, et al. (2008). Association study of variants in two ion-channel genes (TSC and CLCNKB) and hypertension in two ethnic groups in Northwest China. Clin. Chim. Acta 388: 95-98.

Ward NC, Tsai IJ, Barden A, van Bockxmeer FM, et al. (2008). A single nucleotide polymorphism in the CYP4F2 but not CYP4A11 gene is associated with increased 20-HETE excretion and blood pressure. Hypertension 51: 1393-1398.

Zhan YY, Jiang X, Lin G, Li J, et al. (2007). Association of thiazide-sensitive Na+-Cl* cotransporter gene polymorphisms with the risk of essential hypertension. Zhonghua Yi Xue Yi Chuan Xue Za Zhi 24: 703-705. 